\title{
MODELS FOR GROWTH OF HETEROGENEOUS SANDPILES VIA MOSCO CONVERGENCE
}

\author{
M. BOCEA, M. MIHĂILESCU, M. PÉREZ-LLANOS AND J. D. ROSSI
}

\begin{abstract}
In this paper we study the asymptotic behavior of several classes of power-law functionals involving variable exponents $p_{n}(\cdot) \rightarrow \infty$, via Mosco convergence. In the particular case $p_{n}(\cdot)=n p(\cdot)$, we show that the sequence $\left\{H_{n}\right\}$ of functionals $H_{n}: L^{2}\left(\mathbb{R}^{N}\right) \rightarrow[0,+\infty]$ given by

$$
H_{n}(u)= \begin{cases}\int_{\mathbb{R}^{N}} \frac{\lambda(x)^{n}}{n p(x)}|\nabla u(x)|^{n p(x)} d x & \text { if } u \in L^{2}\left(\mathbb{R}^{N}\right) \cap W^{1, n p(\cdot)}\left(\mathbb{R}^{N}\right) \\ +\infty & \text { otherwise }\end{cases}
$$

converges in the sense of Mosco to a functional which vanishes on the set

$$
\left\{u \in L^{2}\left(\mathbb{R}^{N}\right): \lambda(x)|\nabla u|^{p(x)} \leq 1 \text { a.e. } x \in \mathbb{R}^{N}\right\}
$$

and is infinite in its complement. We also provide an example of a sequence of functionals whose Mosco limit cannot be described in terms of the characteristic function of a subset of $L^{2}\left(\mathbb{R}^{N}\right)$.

As an application of our results we obtain a model for the growth of a sandpile in which the allowed slope of the sand depends explicitly on the position in the sample.
\end{abstract}

\section{INTRODUCTION.}

The main results of this paper are concerned with the asymptotic behavior of certain power-law functionals with variable exponents by means of Mosco convergence. This notion of variational convergence, introduced by Umberto Mosco in the 1960's [36], provides an appropriate framework for studying the asymptotic behavior of large classes of variational problems, and has been recognized as a powerful tool for the analysis of important problems in Calculus of Variations, Partial Differential Equations, and their applications [5], [17], [18], [34], [37], [38], [39], [45]. We refer to Section 2 for the definition of Mosco convergence, and to [6] for a detailed introduction to the theory.

The study of power-law functionals with variable exponents and the associated PDEs has received a great deal of attention in recent years. Partial differential equations involving variable exponents became popular during the last decade in relation to applications to elasticity and electrorheological fluids [43], [42], [44]. Meanwhile, the underlying functional analytical tools have been extensively developed and new applications, e.g. to image processing [19], have emerged. For general references on the $p(x)$-Laplacian we refer to [23], which includes a thorough bibliography, and to [33], a seminal paper where many of the basic properties of variable exponent spaces were established. The delicate regularity properties of $p(x)$-harmonic functions have been established in [1] and [2].

Recent results on the asymptotic behavior of power-law functionals are motivated by applications to the study of dielectric breakdown, electrical resistivity, and polycrystal plasticity (see, e.g., [31], [7], [8]).

Key words and phrases. Mosco convergence, power-law functionals, variable exponent spaces, sandpile models. 2000 Mathematics Subject Classification. 49J45, 35K55,46E30, 35Q99. 
The asymptotics in the works just cited is undertaken in the framework of De Giorgi's $\Gamma$-convergence [21], [22].

In the present paper we are interested in applications to models of difussion in sandpiles (which are governed by parabolic problems), and we adopt instead the Mosco convergence as the main tool to study the asymptotic behavior of the functionals involved.

To give an idea of the type of convergence results which we pursue in the sequel, let us consider, for each $n \in \mathbb{N}$, the functionals $H_{n}, H_{\infty}: L^{2}\left(\mathbb{R}^{N}\right) \rightarrow[0,+\infty]$ defined by

$$
H_{n}(u)= \begin{cases}\int_{\mathbb{R}^{N}} \frac{\lambda(x)^{n}}{n p(x)}|\nabla u(x)|^{n p(x)} d x & \text { if } u \in L^{2}\left(\mathbb{R}^{N}\right) \cap W^{1, n p(\cdot)}\left(\mathbb{R}^{N}\right), \\ +\infty & \text { otherwise }\end{cases}
$$

and

$$
H_{\infty}(u)= \begin{cases}0 & \text { if } \lambda(x)|\nabla u(x)|^{p(x)} \leq 1 \text { a.e. } x \in \mathbb{R}^{N}, \\ +\infty & \text { otherwise. }\end{cases}
$$

Here $p: \mathbb{R}^{N} \rightarrow(1, \infty)$ is a bounded function and $\lambda \in L^{\infty}\left(\mathbb{R}^{N}\right)$ is such that $0<\alpha \leq \lambda(x) \leq \beta$ a.e. $x \in \mathbb{R}^{N}$. With this notation, one of our results (see Section 4 for details) can be stated as follows:

$$
H_{n} \text { converges in the sense of Mosco to } H_{\infty} \text {. }
$$

Let us now recall some known results on evolution problems. The limiting behavior as $p \rightarrow \infty$ of solutions to the quasilinear parabolic problem

$$
\begin{cases}v_{p, t}-\Delta_{p} v_{p}=f & \text { in }(0, T) \times \mathbb{R}^{N}, \\ v_{p}(x, 0)=u_{0}(x) & \text { in } \mathbb{R}^{N}\end{cases}
$$

was investigated in [26] (see also [4]). Here, $f$ is nonnegative, and represents a given source term which is interpreted physically as adding material to an evolving system within which mass particles are continually rearranged by diffusion. Let us consider the functional

$$
F_{p}(v):= \begin{cases}\frac{1}{p} \int_{\Omega}|\nabla v(y)|^{p} d y & \text { if } u \in L^{2}\left(\mathbb{R}^{N}\right) \cap W^{1, p}\left(\mathbb{R}^{N}\right), \\ +\infty & \text { if } u \in L^{2}\left(\mathbb{R}^{N}\right) \backslash W^{1, p}\left(\mathbb{R}^{N}\right) .\end{cases}
$$

The problem (1.3) has the standard reformulation

$$
\begin{cases}f-v_{p, t}=\partial F_{p}\left(v_{p}\right) & \text { a.e. } t \in(0, T), \\ v(x, 0)=u_{0}(x) & \text { in } \mathbb{R}^{N}\end{cases}
$$

where $\partial F_{p}$ denotes the subdifferential of $F_{p}$ (see Section 2 for the precise definition).

In [26], assuming that $u_{0}$ is a Lipschitz function with compact support satisfying $\left\|\nabla u_{0}\right\|_{L^{\infty}\left(\mathbb{R}^{N}\right)} \leq 1$, and for $f$ a smooth nonnegative function with compact support in $[0, T] \times \mathbb{R}^{N}$, it is shown that there exists a sequence $p_{i} \rightarrow+\infty$ and a limit function $v_{\infty}$ such that, for each $T>0$,

$$
\begin{cases}v_{p_{i}} \rightarrow v_{\infty} & \text { a.e. and in } L^{2}\left(\mathbb{R}^{N} \times(0, T)\right), \\ D v_{p_{i}} \rightarrow D v_{\infty}, v_{p_{i}, t} \rightarrow v_{\infty, t} & \text { weakly in } L^{2}\left(\mathbb{R}^{N} \times(0, T)\right) .\end{cases}
$$


Moreover, $v_{\infty}$ is a solution to the problem

$$
\begin{cases}f(t)-v_{\infty, t} \in \partial F_{\infty}\left(v_{\infty}(t)\right) & \text { a.e. } t \in(0, T), \\ v_{\infty}(x, 0)=u_{0}(x) & \text { in } \mathbb{R}^{N},\end{cases}
$$

where

$$
F_{\infty}(v)= \begin{cases}0 & \text { if }|\nabla v| \leq 1 \\ +\infty & \text { otherwise }\end{cases}
$$

The limiting problem (1.4) governs the movement of a sandpile, with $v_{\infty}(t, x)$ describing the amount of sand at the point $x$ at time $t$, under the main assumption that the sandpile is stable if the slope is less or equal than one and unstable if not.

The model described above has been extensively studied in [26], [27], [30], [40] and [41] (see also [3], [4], [10], [11], [24] and, for numerical approximations, [28] and [29]). As an application of our Moscoconvergence results we extend this to models which take into account the fact that the admissible slopes may depend explicitly on the spatial location due, e.g., to the presence of heterogeneities (different types of sand at different places in the sandpile). When one considers, for example, the functional $H_{n}$ defined by (1.1) on the Hilbert space $L^{2}\left(\mathbb{R}^{N}\right)$, the associated PDE reads as

$$
\left\{\begin{array}{l}
\left(u_{n}\right)_{t}+\operatorname{div}\left(\lambda(x)^{n}\left|\nabla u_{n}\right|^{n p(x)-2} \nabla u_{n}\right)=f \quad \text { a.e. } t \in(0, T), \\
u_{n}(0)=u_{0} .
\end{array}\right.
$$

In this case, general results from [5] and [16] give

$$
u_{n} \rightarrow u \quad \text { in } C\left([0, T]: L^{2}\left(\mathbb{R}^{N}\right)\right),
$$

where $u$ is the solution to the problem

$$
\left\{\begin{array}{l}
u_{t}+\partial H_{\infty}(u) \ni f \quad \text { a.e. } t \in(0, T), \\
u(0)=u_{0},
\end{array}\right.
$$

and where $H_{\infty}: L^{2}\left(\mathbb{R}^{N}\right) \rightarrow[0,+\infty]$ is given by (1.2). The above limiting problem can be seen as a model for the growth of a sandpile where the critical slope of the sand depends on the spatial location. In fact, note that the pointwise restriction in the definition of $H_{\infty}$ reads as

$$
|\nabla u(x)| \leq\left(\frac{1}{\lambda(x)}\right)^{1 / p(x)}=: A(x) \text { a.e. } x \in \mathbb{R}^{N} .
$$

We refer to Section 6 for some explicit examples of solutions to this evolution problem.

The paper is organized as follows: in Section 2 we collect some preliminary results and introduce the notation which will be used in the sequel; in Section 3, for a general sequence of variable exponents, we prove our first Mosco-convergence result; Section 4 deals with the particular case of variable exponents that will be of interest when studying the heterogeneous sandpile model; Section 5 contains an example of a Mosco limit of power-law functionals which is not given by the characteristic function of a set; finally, in Section 6 we apply our result to analyze the new sandpile model. 


\section{Preliminaries.}

In this section we set up the notation which will be used throughout, and we present some preliminary results. We refer to [23], [33] and the survey [32] for more details. Given $\Omega \subseteq \mathbb{R}^{N}$ and $p: \Omega \rightarrow(1, \infty)$ a function in $L^{\infty}(\Omega)$, the variable exponent Lebesgue space $L^{p(x)}(\Omega)$ is defined as follows:

$$
L^{p(x)}(\Omega):=\left\{u: \Omega \rightarrow \mathbb{R} \text { measurable }: \int_{\Omega}|u(x)|^{p(x)} d x<+\infty\right\},
$$

and it is endowed with the norm

$$
|u|_{p(x)}:=\inf \left\{\tau>0: \int_{\Omega}\left|\frac{u(x)}{\tau}\right|^{p(x)} d x \leq 1\right\} .
$$

The variable exponent Sobolev space $W^{1, p(x)}(\Omega)$ is given by

$$
W^{1, p(x)}(\Omega):=\left\{u \in L^{p(x)}(\Omega):|\nabla u| \in L^{p(x)}(\Omega)\right\},
$$

and

$$
\|u\|:=\inf \left\{\tau>0: \int_{\Omega}\left(\left|\frac{\nabla u(x)}{\tau}\right|^{p(x)}+\left|\frac{u(x)}{\tau}\right|^{p(x)}\right) d x \leq 1\right\} .
$$

is a norm on this space. We denote by $W_{0}^{1, p(x)}(\Omega)$ the closure of $C_{0}^{\infty}(\Omega)$ in $W^{1, p(x)}(\Omega)$.

For any function $p$ as above, we define

$$
p^{-}:=\operatorname{ess} \inf _{x \in \Omega} p(x), \quad \text { and } \quad p^{+}:=\operatorname{ess} \sup _{x \in \Omega} p(x) .
$$

In this paper we will only deal with functions $p$ which satisfy $1<p^{-} \leq p^{+}<\infty$. The following result is well-known (see, e.g., [33]).

Proposition 1. (i) The spaces $\left(L^{p(x)}(\Omega),|\cdot|_{p(x)}\right),\left(W^{1, p(x)}(\Omega),\|\cdot\|\right)$ and $\left(W_{0}^{1, p(x)}(\Omega),\|\cdot\|\right)$ are separable and reflexive Banach spaces.

(ii) Hölder inequality holds:

$$
\int_{\Omega}|u v| d x \leq\left(\frac{1}{p^{-}}+\frac{1}{\left(p^{\prime}\right)^{-}}\right)|u|_{p(x)}|v|_{p^{\prime}(x)}, \quad \forall u \in L^{p(x)}(\Omega), \forall v \in L^{p^{\prime}(x)}(\Omega),
$$

where $p^{\prime}(x):=\frac{p(x)}{p(x)-1}$.

Next, we recall the definition of Mosco-convergence. If $X$ is a metric space, and $\left\{A_{n}\right\}$ is a sequence of subsets of $X$, we define

$$
\liminf _{n \rightarrow \infty} A_{n}:=\left\{x \in X: \exists x_{n} \in A_{n}, x_{n} \rightarrow x\right\} \text {, and } \limsup _{n \rightarrow \infty} A_{n}:=\left\{x \in X: \exists x_{n_{k}} \in A_{n_{k}}, x_{n_{k}} \rightarrow x\right\} .
$$

If $X$ is a normed space, we denote by $s-\lim$ and $w-\lim$ the above limits associated, respectively, to the strong and to the weak topology of $X$.

Definition 1. Let $H$ be a Hilbert space. Given $\Psi_{n}, \Psi: H \rightarrow(-\infty,+\infty]$ convex, lower-semicontinuous functionals, we say that $\Psi_{n}$ converges to $\Psi$ in the sense of Mosco if

$$
w-\limsup _{n \rightarrow \infty} \operatorname{Epi}\left(\Psi_{n}\right) \subset \operatorname{Epi}(\Psi) \subset s-\liminf _{n \rightarrow \infty} \operatorname{Epi}\left(\Psi_{n}\right),
$$


where $\operatorname{Epi}\left(\Psi_{n}\right)$ and $\operatorname{Epi}(\Psi)$ denote the epigraphs of the functionals $\Psi_{n}$ and $\Psi$, defined by

$\operatorname{Epi}\left(\Psi_{n}\right):=\left\{(u, \lambda) \in L^{2}\left(\mathbb{R}^{N}\right) \times \mathbb{R}: \lambda \geq \Psi_{n}(u)\right\}$, and $\operatorname{Epi}(\Psi):=\left\{(u, \lambda) \in L^{2}\left(\mathbb{R}^{N}\right) \times \mathbb{R}: \lambda \geq \Psi(u)\right\}$.

Remark 1. We note that (2.1) is equivalent to the requirement that the following two conditions are simultaneously satisfied:

$$
\forall u \in D(\Psi) \quad \exists u_{n} \in D\left(\Psi_{n}\right): u_{n} \rightarrow u \text { and } \Psi(u) \geq \limsup _{n \rightarrow \infty} \Psi_{n}\left(u_{n}\right) ;
$$

for every subsequence $\left\{n_{k}\right\}, \Psi(u) \leq \liminf _{k} \Psi_{n_{k}}\left(u_{k}\right)$ whenever $u_{k} \rightarrow u$.

Here $D(\Psi):=\{u \in H: \Psi(u)<\infty\}$ and $D\left(\Psi_{n}\right):=\left\{u \in H: \Psi_{n}(u)<\infty\right\}$ denote the domain of $\Psi$ and $\Psi_{n}$, respectively.

\section{A result For general Sequences of VARiable exponents $p_{n}(x)$.}

Consider a sequence $\left\{p_{n}\right\} \subset L^{\infty}\left(\mathbb{R}^{N}\right)$ with $\operatorname{essinf}_{x \in \mathbb{R}^{N}} p_{n}(x)>1$ for each $n \in \mathbb{N}$, satisfying the conditions

$$
p_{n}^{-}:=\operatorname{ess} \inf _{x \in \mathbb{R}^{N}} p_{n}(x) \rightarrow \infty \text { as } n \rightarrow \infty
$$

and

$$
\text { there exists a real constant } \gamma>1 \text { such that } p_{n}^{+}:=\operatorname{ess} \sup _{x \in \mathbb{R}^{N}} p_{n}(x) \leq \gamma p_{n}^{-} \text {for all } n \in \mathbb{N} \text {. }
$$

Let

$$
p_{n}^{\prime}(x):=\frac{p_{n}(x)}{p_{n}(x)-1} .
$$

In particular, (3.1) and (3.2) imply that we have

$$
p_{n}^{\prime+} \rightarrow 1 \text { and }\left(p_{n}^{+}\right)^{\frac{1}{p_{n}^{-}}} \rightarrow 1 \text { as } n \rightarrow \infty .
$$

Let $\mu: \mathbb{R}^{N} \rightarrow[-1 / \sqrt{2}, 1 / \sqrt{2}]$ be a function with the property that there exists $q \geq 1$ such that $\mu \in L^{q}\left(\mathbb{R}^{N}\right)$. We note that such functions exist: $\mu \equiv 0$ and $\mu(x)=\frac{\exp (-|x|)}{\sqrt{2}}$ are just two examples.

For $n \in \mathbb{N}$, consider the functionals $F_{n}: L^{2}\left(\mathbb{R}^{N}\right) \rightarrow[0,+\infty]$ defined by

$$
F_{n}(u)= \begin{cases}\int_{\mathbb{R}^{N}} \frac{1}{p_{n}(x)}\left(\mu(x)^{2}+|\nabla u(x)|^{2}\right)^{p_{n}(x) / 2} d x & \text { if } u \in L^{2}\left(\mathbb{R}^{N}\right) \cap W^{1, p_{n}(\cdot)}\left(\mathbb{R}^{N}\right), \\ +\infty & \text { otherwise }\end{cases}
$$

and $F_{\infty}: L^{2}\left(\mathbb{R}^{N}\right) \rightarrow[0,+\infty]$ defined by

$$
F_{\infty}(u)= \begin{cases}0 & \text { if }|\nabla u(x)| \leq \sqrt{1-\mu(x)^{2}} \text { a.e. } x \in \mathbb{R}^{N}, \\ +\infty & \text { otherwise. }\end{cases}
$$

Theorem 1. The sequence $F_{n}$ converges in the sense of Mosco to $F_{\infty}$. 
Proof. We will first show that (2.2) holds, that is

$$
\forall u \in D\left(F_{\infty}\right) \quad \exists u_{n} \in D\left(F_{n}\right): u_{n} \rightarrow u \text { and } F_{\infty}(u) \geq \limsup _{n \rightarrow \infty} F_{n}\left(u_{n}\right) .
$$

Note that, since $u \in D\left(F_{\infty}\right)$, we have $u \in L^{2}\left(\mathbb{R}^{N}\right)$, with $|\nabla u(x)| \leq \sqrt{1-\mu(x)^{2}}$ a.e. $x \in \mathbb{R}^{N}$ and $F_{\infty}(u)=0$.

We claim that $u \in L^{\infty}\left(\mathbb{R}^{N}\right)$. To see this, first note that by an approximation argument we may assume that $u$ is smooth and that $\|\nabla u\|_{L^{\infty}\left(\mathbb{R}^{N}\right)} \neq 0$ (otherwise $u \equiv c$ and since $u \in L^{2}\left(\mathbb{R}^{N}\right), u \equiv 0$ ). Fix $x_{0} \in \mathbb{R}^{N}$ such that $\left|u\left(x_{0}\right)\right|>\|\nabla u\|_{L^{\infty}\left(\mathbb{R}^{N}\right)}$. If there is no such $x_{0}$, then $|u| \leq\|\nabla u\|_{L^{\infty}\left(\mathbb{R}^{N}\right)}$ in $\mathbb{R}^{N}$, and thus there is nothing to prove. For any $y \in B_{1}\left(x_{0}\right)$, we have

$$
|| u(y)|-| u\left(x_{0}\right)|| \leq\left|u(y)-u\left(x_{0}\right)\right| \leq\|\nabla u\|_{L^{\infty}\left(\mathbb{R}^{N}\right)}\left|y-x_{0}\right| \leq\|\nabla u\|_{L^{\infty}\left(\mathbb{R}^{N}\right)} .
$$

This gives

$$
|u(y)| \geq\left|u\left(x_{0}\right)\right|-\|\nabla u\|_{L^{\infty}\left(\mathbb{R}^{N}\right)} \quad \forall y \in B_{1}\left(x_{0}\right) .
$$

Therefore,

$$
\begin{aligned}
\|u\|_{L^{2}\left(\mathbb{R}^{N}\right)}^{2} \geq \int_{B_{1}\left(x_{0}\right)}|u(y)|^{2} d y & \geq \int_{B_{1}\left(x_{0}\right)}\left(\left|u\left(x_{0}\right)\right|-\|\nabla u\|_{L^{\infty}\left(\mathbb{R}^{N}\right)}\right)^{2} d y \\
& =\left(\left|u\left(x_{0}\right)\right|-\|\nabla u\|_{L^{\infty}\left(\mathbb{R}^{N}\right)}\right)^{2}\left|B_{1}(0)\right| .
\end{aligned}
$$

Thus,

$$
\left|u\left(x_{0}\right)\right| \leq \frac{\|u\|_{L^{2}\left(\mathbb{R}^{N}\right)}}{\left|B_{1}(0)\right|^{1 / 2}}+\|\nabla u\|_{L^{\infty}\left(\mathbb{R}^{N}\right)} .
$$

We conclude that $u \in L^{\infty}\left(\mathbb{R}^{N}\right)$, as claimed.

Next, let $\Phi$ be a smooth function compactly supported in $B_{2}(0)$ such that $0<\Phi<1$ in $B_{2}(0) \backslash B_{1}(0)$, $\Phi \equiv 1$ in $B_{1}(0)$ and $\|\nabla \Phi\|_{\infty} \leq C$. For each $n \in \mathbb{N}$, define

$$
\varphi_{n}(x)=\left(1-\varepsilon_{n}\right) \Phi\left(\frac{x}{R_{n}}\right)
$$

with $R_{n}$ and $\varepsilon_{n}$ to be chosen later, satisfying

$$
R_{n} \rightarrow \infty \text { and } \varepsilon_{n} \rightarrow 0 \text { as } n \rightarrow \infty
$$

Let us now define the sequence $v_{n}:=u \varphi_{n}$. It is clear that $v_{n} \in D\left(F_{n}\right)$ and, in view of (3.4),

$$
\begin{gathered}
\int_{\mathbb{R}^{N}}\left|u-v_{n}\right|^{2} d x=\int_{\mathbb{R}^{N} \backslash B_{R_{n}}(0)}\left|u-v_{n}\right|^{2} d x+\int_{B_{R_{n}}(0)}\left|u-v_{n}\right|^{2} d x \\
\leq 4 \int_{\mathbb{R}^{N} \backslash B_{R_{n}}(0)}|u|^{2} d x+\varepsilon_{n}^{2} \int_{B_{R_{n}}(0)}|u|^{2} d x \rightarrow 0 \text { as } n \rightarrow \infty .
\end{gathered}
$$

On the other hand,

$$
\left|\nabla v_{n}(x)\right|=\left|\nabla u(x) \varphi_{n}(x)+u(x) \nabla \varphi_{n}(x)\right|=\left|\nabla u(x)\left(1-\varepsilon_{n}\right) \Phi\left(\frac{x}{R_{n}}\right)+u(x)\left(1-\varepsilon_{n}\right) \nabla \Phi\left(\frac{x}{R_{n}}\right) \frac{1}{R_{n}}\right| .
$$


Therefore, using the fact that $\nabla \Phi=0$ in $B_{R_{n}}(0)$, and since $0 \leq \Phi \leq 1,|\nabla \Phi| \leq C$ in the whole $\mathbb{R}^{N}$, $|\nabla u| \leq \sqrt{1-\mu^{2}} \leq 1$ a.e. in $\mathbb{R}^{N}$, we get

$$
\left|\nabla v_{n}(x)\right| \leq\left(1-\varepsilon_{n}\right) \chi_{B_{R_{n}}(0)}(x)+\left(\left(1-\varepsilon_{n}\right)+C\|u\|_{L^{\infty}\left(\mathbb{R}^{N}\right)} \frac{\left(1-\varepsilon_{n}\right)}{R_{n}}\right) \chi_{B_{2 R_{n}}(0) \backslash B_{R_{n}}(0)}(x) .
$$

Hence,

$$
\left|\nabla v_{n}(x)\right|^{p_{n}(x)} \leq 1 \chi_{B_{R_{n}}(0)}(x)+1 \chi_{B_{2 R_{n}}(0) \backslash B_{R_{n}}(0)}(x),
$$

provided that we choose $R_{n} \rightarrow \infty$ and $\varepsilon_{n} \rightarrow 0$ such that

$$
\left(\left(1-\varepsilon_{n}\right)+C\|u\|_{L^{\infty}\left(\mathbb{R}^{N}\right)} \frac{\left(1-\varepsilon_{n}\right)}{R_{n}}\right) \leq 1 .
$$

This can be achieved if

$$
\varepsilon_{n} \geq C\|u\|_{L^{\infty}\left(\mathbb{R}^{N}\right)} \frac{\left(1-\varepsilon_{n}\right)}{R_{n}},
$$

which clearly holds for $n \in \mathbb{N}$ large enough if we impose (in addition to (3.4)) that

$$
\varepsilon_{n} R_{n} \rightarrow \infty \text { as } n \rightarrow \infty \text {. }
$$

Taking into account the previous estimates, and since by (3.1) we have $\max \{4, q\}<p_{n}^{-}$for $n \in \mathbb{N}$ sufficiently large, we get

$$
\begin{aligned}
F_{n}\left(v_{n}\right) & =\int_{\mathbb{R}^{N}} \frac{1}{p_{n}(x)}\left(\mu(x)^{2}+\left|\nabla v_{n}(x)\right|^{2}\right)^{p_{n}(x) / 2} d x \leq \int_{\mathbb{R}^{N}} \frac{1}{p_{n}(x)}\left(\mu(x)^{2}+\chi_{B_{2 R_{n}}(0)}(x)\right)^{p_{n}(x) / 2} d x \\
& \leq \int_{\mathbb{R}^{N}} \frac{1}{p_{n}(x)} 2^{p_{n}(x) / 2}\left(|\mu(x)|^{p_{n}(x)}+\chi_{B_{2 R_{n}}(0)}(x)\right) d x \\
& \leq \frac{1}{p_{n}^{-}} \int_{\mathbb{R}^{N}}(\sqrt{2}|\mu(x)|)^{p_{n}(x)} d x+\int_{B_{2 R_{n}}(0)} \frac{1}{p_{n}(x)} d x \\
& \leq \frac{1}{p_{n}^{-}} \int_{\mathbb{R}^{N}}(\sqrt{2}|\mu(x)|)^{q} d x+C \frac{R_{n}^{N}}{p_{n}^{-}} \rightarrow 0 \text { as } n \rightarrow \infty,
\end{aligned}
$$

provided that we choose $R_{n}$ such that

$$
\frac{R_{n}^{N}}{p_{n}^{-}} \rightarrow 0 \text { as } n \rightarrow \infty \text {. }
$$

This shows that $\limsup _{n \rightarrow \infty} F_{n}\left(v_{n}\right) \leq 0=F_{\infty}(u)$, and thus (2.2) holds. Now, observe that the choices

$$
R_{n}:=\left(p_{n}^{-}\right)^{\frac{1}{2 N}} \quad \text { and } \quad \varepsilon_{n}:=\left(p_{n}^{-}\right)^{-\frac{1}{4 N}},
$$

ensure that (3.4), (3.5), and (3.6) are satisfied.

It remains to show that, whenever $u_{n_{k}} \in D\left(F_{n_{k}}\right)$ is such that $u_{n_{k}} \rightarrow u$ weakly in $L^{2}\left(\mathbb{R}^{N}\right)$ as $k \rightarrow \infty$, then $\liminf _{k \rightarrow \infty} F_{n_{k}}\left(u_{n_{k}}\right) \geq F_{\infty}(u)$.

To proceed further, we cover $\mathbb{R}^{N}$ with a countable number of open and bounded domains $\Omega$ (not necessary disjoint) with $|\Omega|=1$. Fix such an $\Omega$. Let $x \in \Omega$ be a Lebesgue point for $\sqrt{\lambda^{2}+|\nabla u|^{2}} \in$ $L^{1}(\Omega)$. Then, for each $r>0$ sufficiently small, we have $B_{r}(x) \subset \Omega$.

For each open set $\omega \subset \mathbb{R}^{N}$ and each $n_{k} \in \mathbb{N}$, define

$$
\omega_{n_{k}, \mu}^{+}:=\left\{x \in \omega ; \sqrt{\mu(x)^{2}+\left|\nabla u_{n_{k}}(x)\right|^{2}}>1\right\} \text { and } \omega_{n_{k}, \mu}^{-}:=\left\{x \in \omega ; \sqrt{\mu(x)^{2}+\left|\nabla u_{n_{k}}(x)\right|^{2}} \leq 1\right\} \text {. }
$$


We have

$$
\begin{aligned}
& \int_{B_{r}(x)} \sqrt{|\mu(y)|^{2}+\left|\nabla u_{n_{k}}(y)\right|^{2}} d y=\int_{\left(B_{r}(x)\right)_{n_{k}, \mu}^{+}} \sqrt{|\mu(y)|^{2}+\left|\nabla u_{n_{k}}(y)\right|^{2}} d y \\
& +\int_{\left.\left(B_{r}(x)\right)\right)_{n_{k}, \mu}^{-}} \sqrt{|\mu(y)|^{2}+\left|\nabla u_{n_{k}}(y)\right|^{2}} d y \\
& \leq\left(\int_{\left(B_{r}(x)\right)_{n_{k}, \mu}^{+}}\left(|\mu(y)|^{2}+\left|\nabla u_{n_{k}}(y)\right|^{2}\right)^{p_{n_{k}}^{-} / 2} d y\right)^{\frac{1}{p_{n_{k}}^{-}}}\left|\left(B_{r}(x)\right)_{n_{k}, \mu}^{+}\right|^{\frac{p_{n_{k}}^{-}-1}{p_{n_{k}}^{-}}} \\
& +\left(\int_{\left(B_{r}(x)\right)_{n_{k}, \mu}^{-}}\left(|\mu(y)|^{2}+\left|\nabla u_{n_{k}}(y)\right|^{2}\right)^{p_{n_{k}}^{+} / 2} d y\right)^{\frac{1}{p_{n_{k}}^{+}}}\left|\left(B_{r}(x)\right)_{n_{k}, \mu}^{-}\right|^{\frac{p_{n_{k}}^{+}-1}{p_{n_{k}}^{+}}} \\
& \leq\left(p_{n_{k}}^{+} \int_{\left(B_{r}(x)\right)_{n_{k}, \mu}^{+}} \frac{1}{p_{n_{k}}(y)}\left(|\mu(y)|^{2}+\left|\nabla u_{n_{k}}(y)\right|^{2}\right)^{p_{n_{k}}(y) / 2} d y\right)^{\frac{1}{p_{n_{k}}^{-}}}\left|\left(B_{r}(x)\right)_{n_{k}, \mu}^{+}\right|^{\frac{p_{n_{k}}^{-}-1}{p_{n_{k}}^{-}}} \\
& +\left(p_{n_{k}}^{+} \int_{\left(B_{r}(x)\right)_{n_{k}, \mu}^{-}} \frac{1}{p_{n_{k}}(y)}\left(|\mu(y)|^{2}+\left|\nabla u_{n_{k}}(y)\right|^{2}\right)^{p_{n_{k}}(y) / 2} d y\right)^{\frac{1}{p_{n_{k}}^{+}}}\left|\left(B_{r}(x)\right)_{n_{k}, \mu}^{-}\right|^{\frac{p_{n_{k}}^{+}-1}{p_{n_{k}}^{+}}} \\
& \leq\left(p_{n_{k}}^{+} F_{n_{k}}\left(u_{n_{k}}\right)\right)^{\frac{1}{p_{n_{k}}^{-}}}\left|\left(B_{r}(x)\right)_{n_{k}, \mu}^{+}\right|^{\frac{p_{n_{k}}^{-}-1}{p_{n_{k}}^{-}}}+\left(p_{n_{k}}^{+} F_{n_{k}}\left(u_{n_{k}}\right)\right)^{\frac{1}{p_{n_{k}}^{+}}}\left|\left(B_{r}(x)\right)_{n_{k}, \mu}^{-}\right|^{\frac{p_{n_{k}}^{+}-1}{p_{n_{k}}^{+}}} .
\end{aligned}
$$

Since

$$
\limsup _{k \rightarrow \infty}\left(p_{n_{k}}^{+} F_{n_{k}}\left(u_{n_{k}}\right)\right)^{\frac{1}{p_{n_{k}}^{-}}} \leq 1 \text { and } \limsup _{k \rightarrow \infty}\left(p_{n_{k}}^{+} F_{n_{k}}\left(u_{n_{k}}\right)\right)^{\frac{1}{p_{n_{k}}^{+}}} \leq 1
$$

we obtain that

$$
\limsup _{k \rightarrow \infty}\left(\left(p_{n_{k}}^{+} F_{n_{k}}\left(u_{n_{k}}\right)\right)^{\frac{1}{p_{n_{k}}^{-}}}\left|\left(B_{r}(x)\right)_{n_{k}, \mu}^{+}\right|^{\frac{p_{n_{k}}^{-}-1}{p_{n_{k}}}}+\left(p_{n_{k}}^{+} F_{n_{k}}\left(u_{n_{k}}\right)\right)^{\frac{1}{p_{n_{k}}^{+}}}\left|\left(B_{r}(x)\right)_{n_{k}, \mu}^{-}\right|^{\frac{p_{n_{k}}^{+}-1}{p_{n_{k}}^{+}}}\right) \leq\left|B_{r}(x)\right|,
$$

and hence

$$
\limsup _{k \rightarrow \infty} \int_{B_{r}(x)} \sqrt{|\mu(y)|^{2}+\left|\nabla u_{n_{k}}(y)\right|^{2}} d y \leq\left|B_{r}(x)\right| .
$$

In view of (3.1), we have that $2<p_{n_{k}}^{-}$for sufficiently large $k \in \mathbb{N}$. Thus, using the classical Hölder's inequality we deduce that

$$
\begin{aligned}
\int_{\Omega}\left|\nabla u_{n_{k}}(x)\right|^{2} d x & \leq\left(\int_{\Omega}\left|\nabla u_{n_{k}}(x)\right|^{p_{n_{k}}^{-}} d x\right)^{\frac{2}{p_{n_{k}}^{-}}}|\Omega|^{\frac{p_{n_{k}}^{-}-2}{p_{n_{k}}^{-}}} \\
& =\left(\int_{\Omega_{n_{k}, 0}^{-}}\left|\nabla u_{n_{k}}(x)\right|^{p_{n_{k}}^{-}} d x+\int_{\Omega_{n_{k}, 0}^{+}}\left|\nabla u_{n_{k}}(x)\right|^{p_{n_{k}}^{-}} d x\right)^{\frac{2}{p_{n_{k}}^{-}}} \\
& \leq\left(1+\int_{\Omega}\left|\nabla u_{n_{k}}(x)\right|^{p_{n_{k}}(x)} d x\right)^{\frac{2}{p_{n_{k}}^{-}}} \leq\left(1+p_{n_{k}}^{+} F_{n_{k}}\left(u_{n_{k}}\right)\right)^{\frac{2}{p_{n_{k}}^{-}}} .
\end{aligned}
$$

It follows that the sequence $\left\{\nabla u_{n_{k}}\right\}$ is bounded in $L^{2}\left(\Omega ; \mathbb{R}^{N}\right)$. Since $u_{n_{k}} \rightarrow u$ weakly in $L^{2}\left(\mathbb{R}^{N}\right)$, we deduce that $\left\{u_{n_{k}}\right\}$ is bounded in $L^{2}(\Omega)$. Overall, $\left\{u_{n_{k}}\right\}$ is bounded in $W^{1,2}(\Omega)$, and thus we may 
extract a subsequence (not relabelled) such that $u_{n_{k}} \rightarrow u$ weakly in $W^{1,2}(\Omega)$. Using a well-known lower semicontinuity result we find

$$
\int_{B_{r}(x)} \sqrt{|\mu(y)|^{2}+|\nabla u(y)|^{2}} d y \leq \liminf _{k \rightarrow \infty} \int_{B_{r}(x)} \sqrt{|\mu(y)|^{2}+\left|\nabla u_{n_{k}}(y)\right|^{2}} d y
$$

which implies, in view of (3.7), that

$$
\frac{1}{\left|B_{r}(x)\right|} \int_{B_{r}(x)} \sqrt{|\mu(y)|^{2}+|\nabla u(y)|^{2}} d y \leq 1 .
$$

Since almost every $x \in \Omega$ is a Lebesgue point for $\sqrt{\lambda^{2}+|\nabla u|^{2}}$, passing to the limit $r \rightarrow 0^{+}$in the above inequality yields $\sqrt{|\mu(x)|^{2}+|\nabla u(x)|^{2}} \leq 1$ for a.e. $x \in \Omega$, that is, $|\nabla u(x)| \leq \sqrt{1-\mu(x)^{2}}$ for a.e. $x \in \Omega$.

Since $\mathbb{R}^{N}$ is covered by a countable number of sets of type $\Omega$ it follows that $|\nabla u(x)| \leq \sqrt{1-\mu(x)^{2}}$ for a.e. $x \in \mathbb{R}^{N}$. Hence, $F_{\infty}(u)=0$, and we deduce that $\liminf _{k \rightarrow \infty} F_{n_{k}}\left(u_{n_{k}}\right) \geq F_{\infty}(u)$. This concludes the proof of Theorem 1.

Remark 2. The limiting functional in Theorem 1 coincides with the one obtained in the case of constant exponents, $p_{n}(x)=n$. Hence, in this case, the presence of the variable exponents does not induce any particular spatial dependence in the limit. This fact is even more transparent if we focus our attention on the particular case where $\mu \equiv 0$ : the functionals $F_{n}$ are then given by

$$
\begin{cases}\int_{\mathbb{R}^{N}} \frac{1}{p_{n}(x)}|\nabla u(x)|^{p_{n}(x)} d x & \text { if } u \in L^{2}\left(\mathbb{R}^{N}\right) \cap W^{1, p_{n}(\cdot)}\left(\mathbb{R}^{N}\right), \\ +\infty & \text { otherwise }\end{cases}
$$

and the limiting functional $F_{\infty}$ becomes

$$
\begin{cases}0 & \text { if }|\nabla u(x)| \leq 1 \text { a.e. } x \in \mathbb{R}^{N}, \\ +\infty & \text { otherwise. }\end{cases}
$$

This observation is the starting point for the study undertaken in the next section.

\section{THE CASE $p_{n}(x)=n p(x)$.}

In this section we consider the particular case $p_{n}(x)=n p(x)$, and we work with a sequence of functionals which will allow us to obtain a more involved dependence on $x$ in the Mosco limit.

Theorem 2. Let $p: \mathbb{R}^{N} \rightarrow(1, \infty)$ be a bounded function, and let $\lambda \in L^{\infty}\left(\mathbb{R}^{N}\right), 0<\alpha \leq \lambda(x) \leq \beta$ for a.e. $x \in \mathbb{R}^{N}$. For each $n \in \mathbb{N}$, consider the functionals $H_{n}, H_{\infty}: L^{2}\left(\mathbb{R}^{N}\right) \rightarrow[0,+\infty]$ defined by

$$
H_{n}(u)= \begin{cases}\int_{\mathbb{R}^{N}} \frac{\lambda(x)^{n}}{n p(x)}|\nabla u(x)|^{n p(x)} d x & \text { if } u \in L^{2}\left(\mathbb{R}^{N}\right) \cap W^{1, n p(\cdot)}\left(\mathbb{R}^{N}\right), \\ +\infty & \text { otherwise, }\end{cases}
$$

and

$$
H_{\infty}(u)= \begin{cases}0 & \text { if } \lambda(x)|\nabla u(x)|^{p(x)} \leq 1 \text { a.e. } x \in \mathbb{R}^{N}, \\ +\infty & \text { otherwise. }\end{cases}
$$

Then $H_{n}$ converges in the sense of Mosco to $H_{\infty}$. 
Proof. We divide the proof into two parts.

I. First, we show that

$$
\forall u \in D\left(H_{\infty}\right) \quad \exists u_{n} \in D\left(H_{n}\right): u_{n} \rightarrow u \text { and } H_{\infty}(u) \geq \limsup _{n \rightarrow \infty} H_{n}\left(u_{n}\right) .
$$

Let $u \in D\left(H_{\infty}\right)$, that is, $u \in L^{2}\left(\mathbb{R}^{N}\right)$ and $\lambda(x)|\nabla u(x)|^{p(x)} \leq 1$ a.e. $x \in \mathbb{R}^{N}$, in which case $H_{\infty}(u)=0$. We proceed as in the proof of Theorem 1 . Let $\left\{\varepsilon_{n}\right\},\left\{R_{n}\right\}$ be the two sequences defined by

$$
R_{n}:=\left(n p^{-}\right)^{\frac{1}{2 N}} \quad \text { and } \quad \varepsilon_{n}:=\left(n p^{-}\right)^{-\frac{1}{4 N}},
$$

so that (3.4), (3.5), and (3.6) hold. Further, let $\Phi, \varphi_{n}$ be as in the proof of Theorem 1, and define $u_{n} \in L^{2}\left(\mathbb{R}^{N}\right) \cap W^{1, n p(\cdot)}\left(\mathbb{R}^{N}\right)$ by $u_{n}:=u \varphi_{n}$. Then, $u_{n} \rightarrow u$ in $L^{2}\left(\mathbb{R}^{n}\right)$, and we have

$$
\begin{aligned}
\lambda(x)^{\frac{1}{p(x)}}\left|\nabla u_{n}(x)\right| & =\left|\lambda(x)^{\frac{1}{p(x)}} \nabla u(x) \varphi_{n}(x)+\lambda(x)^{\frac{1}{p(x)}} u(x) \nabla \varphi_{n}(x)\right| \\
& =\left|\lambda(x)^{\frac{1}{p(x)}} \nabla u(x)\left(1-\varepsilon_{n}\right) \Phi\left(\frac{x}{R_{n}}\right)+\lambda(x)^{\frac{1}{p(x)}} u(x)\left(1-\varepsilon_{n}\right) \nabla \Phi\left(\frac{x}{R_{n}}\right) \frac{1}{R_{n}}\right| .
\end{aligned}
$$

Since $\nabla \Phi=0$ in $B_{R_{n}}(0), 0 \leq \Phi \leq 1,|\nabla \Phi| \leq C$, and $\lambda(x)^{\frac{1}{p(x)}}|\nabla u(x)| \leq 1$ a.e. $x \in \mathbb{R}^{N}$, we deduce that there exists a constant $C_{1}>0$ such that

$$
\lambda(x)^{\frac{1}{p(x)}}\left|\nabla u_{n}(x)\right| \leq\left(1-\varepsilon_{n}\right) \chi_{B_{R_{n}}(0)}(x)+\left(\left(1-\varepsilon_{n}\right)+C_{1}|u|_{\infty} \frac{\left(1-\varepsilon_{n}\right)}{R_{n}}\right) \chi_{B_{2 R_{n}}(0) \backslash B_{R_{n}}(0)}(x) .
$$

Since $\varepsilon_{n} \rightarrow 0$ and $\varepsilon_{n} R_{n} \rightarrow \infty$ as $n \rightarrow \infty$ we have, for $n \in \mathbb{N}$ sufficiently large,

$$
\left(1-\varepsilon_{n}\right)+C_{1}|u|_{\infty} \frac{\left(1-\varepsilon_{n}\right)}{R_{n}} \leq 1
$$

Hence,

$$
\lambda(x)\left|\nabla u_{n}(x)\right|^{p(x)} \leq \chi_{B_{2 R_{n}}(0)}(x) \text { a.e. } x \in \mathbb{R}^{N} .
$$

Thus, there exists a constant $C_{2}>0$ such that, for $n \in \mathbb{N}$ sufficiently large,

$$
H_{n}\left(u_{n}\right)=\int_{\mathbb{R}^{N}} \frac{\lambda(x)^{n}}{n p(x)}\left|\nabla u_{n}(x)\right|^{n p(x)} d x \leq \int_{B_{2 R_{n}}(0)} \frac{1}{n p(x)} d x \leq C_{2} \frac{R_{n}^{N}}{n p^{-}}=C_{2} \frac{1}{\sqrt{n p^{-}}} .
$$

We deduce that $\lim \sup H_{n}\left(u_{n}\right)=0=H_{\infty}(u)$.

II. Let $u \in L^{2}\left(\mathbb{R}^{N}\right)$. We will show that $H_{\infty}(u) \leq \liminf _{n \rightarrow \infty} H_{n}\left(u_{n}\right)$ whenever $\left\{u_{n}\right\} \subset L^{2}\left(\mathbb{R}^{N}\right)$ is such that $u_{n} \rightarrow u$ weakly in $L^{2}\left(\mathbb{R}^{N}\right)$.

We may assume, without loss of generality, that $u_{n} \in L^{2}\left(\mathbb{R}^{N}\right) \cap W^{1, n p(\cdot)}\left(\mathbb{R}^{N}\right)$, and that we have

$$
\liminf _{n \rightarrow \infty} H_{n}\left(u_{n}\right)=\lim _{n \rightarrow \infty} H_{n}\left(u_{n}\right)<+\infty .
$$

Let $\left\{\Omega_{j}: j=1,2, \cdots\right\}$ be a collection of open sets with sufficiently smooth boundaries such that $\left|\Omega_{j}\right|=1 \forall j \in \mathbb{N}$, and $\bigcup_{j=1}^{n} \Omega_{j}=\mathbb{R}^{N}$. Fix $j \in \mathbb{N}$, and let $q \geq 2$ be arbitrary. For $n \in \mathbb{N}$ sufficiently large, we have

$$
\int_{\Omega_{j}}\left|\nabla u_{n}(x)\right|^{q} d x \leq\left.\left. 2|| \nabla u_{n}\right|^{q}\right|_{n p(\cdot) / q}
$$


and note that if $\left.\left.|| \nabla u_{n}\right|^{q}\right|_{n p(\cdot) / q}>1$, we obtain

$$
\left.\left.|| \nabla u_{n}\right|^{q}\right|_{n p(\cdot) / q} ^{n p^{-} / q} \leq \int_{\Omega_{j}}\left|\nabla u_{n}(x)\right|^{n p(x)} d x \leq \frac{n p^{+}}{\alpha^{n}} \int_{\Omega_{j}} \frac{\lambda(x)^{n}}{n p(x)}\left|\nabla u_{n}(x)\right|^{n p(x)} d x \leq \frac{n p^{+}}{\alpha^{n}} H_{n}\left(u_{n}\right) .
$$

Thus,

$$
\|\left.\left.\nabla u_{n}\right|^{q}\right|_{n p(\cdot) / q} \leq \max \left\{1,\left(\frac{n p^{+}}{\alpha^{n}}\right)^{q / n p^{-}} H_{n}\left(u_{n}\right)^{q / n p^{-}}\right\} .
$$

In view of (4.2) and (4.3) we deduce that $\left\{\nabla u_{n}\right\}$ is bounded in $L^{q}\left(\Omega_{j} ; \mathbb{R}^{N}\right)$. Since $u_{n} \rightarrow u$ weakly in $L^{2}\left(\mathbb{R}^{N}\right)$ and $q \geq 2$ we have that the sequence $\left\{u_{n}\right\}$ is bounded in $L^{q}\left(\Omega_{j}\right)$. Hence, $\left\{u_{n}\right\}$ is bounded in $W^{1, q}\left(\Omega_{j}\right)$, and we may extract a subsequence (not relabelled), such that $u_{n} \rightarrow u$ weakly in $W^{1, q}\left(\Omega_{j}\right)$. In particular, for each $j \in \mathbb{N}$ fixed, we have $\nabla u \in L^{q}\left(\Omega_{j} ; \mathbb{R}^{N}\right)$ for all $q \geq 2$. Thus, since $\lambda \in L^{\infty}\left(\mathbb{R}^{N}\right)$, we obtain that $\lambda(\cdot)|\nabla u(\cdot)|^{p(\cdot)} \in L^{1}\left(\Omega_{j}\right)$. Let $x \in \Omega_{j}$ be a Lebesgue point for this map, and let $r>0$ be small enough so that $B_{r}(x) \subset \Omega_{j}$. We have (by arguments similar to those following (5.7) in the proof of Theorem 3 in the next section)

$$
\int_{B_{r}(x)} \lambda(y)|\nabla u(y)|^{p(y)} d y \leq \liminf _{n \rightarrow \infty} \int_{B_{r}(x)} \lambda(y)\left|\nabla u_{n}(y)\right|^{p(y)} d y .
$$

On the other hand,using Hölder's inequality,

$$
\begin{aligned}
\int_{B_{r}(x)} \lambda(y)\left|\nabla u_{n}(y)\right|^{p(y)} d y & \leq\left\|\lambda(\cdot)\left|\nabla u_{n}(\cdot)\right|^{p(\cdot)}\right\|_{L^{n}\left(B_{r}(x)\right)}\left|B_{r}(x)\right|^{(n-1) / n} \\
& \leq\left(n p^{+} \int_{B_{r}(x)} \frac{\lambda(y)^{n}}{n p(y)}|\nabla u(y)|^{n p(y)} d y\right)^{1 / n}\left|B_{r}(x)\right|^{(n-1) / n} \\
& \leq\left(n p^{+} H_{n}\left(u_{n}\right)\right)^{1 / n}\left|B_{r}(x)\right|^{(n-1) / n} .
\end{aligned}
$$

Passing to the limit as $n \rightarrow \infty$ we deduce that

$$
\limsup _{n \rightarrow \infty} \int_{B_{r}(x)} \lambda(y)\left|\nabla u_{n}(y)\right|^{p(y)} d y \leq\left|B_{r}(x)\right| .
$$

Taking into account (4.4), we obtain

$$
\frac{1}{\left|B_{r}(x)\right|} \int_{B_{r}(x)} \lambda(y)|\nabla u(y)|^{p(y)} d y \leq 1
$$

Since $x \in \Omega_{j}$ was a Lebesgue point for $\lambda(\cdot)|\nabla u(\cdot)|^{p(\cdot)}$, we have that $\lambda(x)|\nabla u(x)|^{p(x)} \leq 1$. Thus, this inequality holds for a.e. $x \in \Omega_{j}(j=1,2, \cdots)$. Since $\left\{\Omega_{j}\right\}_{j \in \mathbb{N}}$ is a countable covering of $\mathbb{R}^{N}$, we deduce that $\lambda(x)|\nabla u(x)|^{p(x)} \leq 1$ for a.e. $x \in \mathbb{R}^{N}$. We conclude that $H_{\infty}(u)=0$, and thus

$$
H_{\infty}(u) \leq \liminf _{n \rightarrow \infty} H_{n}\left(u_{n}\right)
$$

This concludes the proof. 


\section{An example of a nondegenerate Mosco limit}

The aim of our next result is to show that a suitable scaling of our previous energy (with $\lambda \equiv 1$ ) gives rise to a nondegenerate Mosco-limit.

Theorem 3. Let $p: \mathbb{R}^{N} \rightarrow(1, \infty)$ be a bounded function. For $n \in \mathbb{N}$, consider the functionals $G_{n}, G_{\infty}: L^{2}\left(\mathbb{R}^{N}\right) \rightarrow[0,+\infty]$ defined by

$$
G_{n}(u)= \begin{cases}\left(\int_{\mathbb{R}^{N}} \frac{1}{n p(x)}|\nabla u(x)|^{n p(x)} d x\right)^{1 / n} & \text { if } u \in L^{2}\left(\mathbb{R}^{N}\right) \cap W^{1, n p(\cdot)}\left(\mathbb{R}^{N}\right), \\ +\infty & \text { otherwise, }\end{cases}
$$

and

$$
G_{\infty}(u)= \begin{cases}\left\||\nabla u|^{p(\cdot)}\right\|_{L^{\infty}\left(\mathbb{R}^{N}\right)} & \text { if } \nabla u \in L^{\infty}\left(\mathbb{R}^{N} ; \mathbb{R}^{N}\right) \\ +\infty & \text { otherwise. }\end{cases}
$$

Then $G_{n}$ converges in the sense of Mosco to $G_{\infty}$, i.e. the following hold:

$$
\forall u \in D\left(G_{\infty}\right) \quad \exists u_{n} \in D\left(G_{n}\right): u_{n} \rightarrow u \text { and } G_{\infty}(u) \geq \limsup _{n \rightarrow \infty} G_{n}\left(u_{n}\right)
$$

(5.2) for every subsequence $\left\{n_{k}\right\}, G_{\infty}(u) \leq \liminf _{k \rightarrow \infty} G_{n_{k}}\left(u_{k}\right)$ whenever $u_{k} \rightarrow u$ weakly in $L^{2}\left(\mathbb{R}^{N}\right)$.

Proof. I. We show first that (5.1) holds. Let $u \in D\left(G_{\infty}\right)$. Then, $G_{\infty}(u)=\left\||\nabla u|^{p(\cdot)}\right\|_{L^{\infty}\left(\mathbb{R}^{N}\right)}$ and $u \in L^{2}\left(\mathbb{R}^{N}\right),|\nabla u| \in L^{\infty}\left(\mathbb{R}^{N}\right)$. A similar proof to the one given in Theorem 1 shows that we can obtain estimates for $|u(x)|$ in terms of $\|u\|_{L^{2}\left(\mathbb{R}^{N}\right)}$ and $\||\nabla u|\|_{L^{\infty}\left(\mathbb{R}^{N}\right)}$. Next, let $\Phi, \varepsilon_{n}, R_{n}, \varphi_{n}, v_{n}$ be defined as in the proof of Theorem 1 . We have $v_{n} \in L^{2}\left(\mathbb{R}^{N}\right) \cap W^{1, n p(\cdot)}\left(\mathbb{R}^{N}\right)=D\left(G_{n}\right)$, and the same proof as in Theorem 1 shows that $v_{n} \rightarrow u$ in $L^{2}\left(\mathbb{R}^{N}\right)$.

It remains to show that $G_{\infty}(u) \geq \limsup _{n \rightarrow \infty} G_{n}\left(v_{n}\right)$. To this aim, we establish first the following: for any $\varepsilon>0$ there exists $\delta(\varepsilon)>0$ such that

$$
(1+t)^{p(x)}-t^{p(x)}<\varepsilon t^{p(x)}+(1+\delta(\varepsilon))^{p^{+}}, \quad \forall x \in \mathbb{R}^{N}, \forall t \geq 0 .
$$

Let $\varepsilon>0$ be fixed. Since

$$
\lim _{t \rightarrow \infty}\left[\left(1+\frac{1}{t}\right)^{p^{+}}-1\right]=0,
$$

it follows that there exists $\delta(\varepsilon)>0$ such that

$$
\left(1+\frac{1}{t}\right)^{p^{+}}-1<\varepsilon, \quad \forall t>\delta(\varepsilon)
$$

Hence

$$
\left(1+\frac{1}{t}\right)^{p(x)}-1<\varepsilon, \quad \forall t>\delta(\varepsilon), \forall x \in \mathbb{R}^{N},
$$

or, equivalently,

$$
(1+t)^{p(x)}-t^{p(x)}<\varepsilon t^{p(x)}, \quad \forall t>\delta(\varepsilon), \forall x \in \mathbb{R}^{N} .
$$

On the other hand, for any $x \in \mathbb{R}^{N}$ and any $t \in[0, \delta(\varepsilon)]$ we have

$$
(1+t)^{p(x)}-t^{p(x)}<(1+t)^{p^{+}}<(1+\delta(\varepsilon))^{p^{+}} .
$$


The last two inequalities show that (5.3) holds. Furthermore, (5.3) implies that for any $\varepsilon>0$ there exists $\delta(\varepsilon)>0$ such that

$$
(a+b)^{p(x)}<(\varepsilon+1) a^{p(x)}+(1+\delta(\varepsilon))^{p^{+}} b^{p(x)}, \quad \forall x \in \mathbb{R}^{N}, \forall a, b \geq 0 .
$$

Now, the same computations as in the proof of Theorem 1 yield that for each $x \in \mathbb{R}^{N}$ we have

$$
\left|\nabla v_{n}(x)\right|=\left|\nabla u(x) \varphi_{n}(x)+u(x) \nabla \varphi_{n}(x)\right|=\left|\nabla u(x)\left(1-\varepsilon_{n}\right) \Phi\left(\frac{x}{R_{n}}\right)+u(x)\left(1-\varepsilon_{n}\right) \nabla \Phi\left(\frac{x}{R_{n}}\right) \frac{1}{R_{n}}\right|,
$$

and it follows that

$$
\left|\nabla v_{n}(x)\right| \leq|\nabla u(x)|\left(1-\varepsilon_{n}\right) \chi_{B_{2 R_{n}}(0)}+C\|u\|_{L^{\infty}\left(\mathbb{R}^{N}\right)} \frac{\left(1-\varepsilon_{n}\right)}{R_{n}} \chi_{B_{2 R_{n}}(0) \backslash B_{R_{n}}(0)} .
$$

Thus,

$$
\left|\nabla v_{n}(x)\right|^{p(x)} \leq\left(|\nabla u(x)|\left(1-\varepsilon_{n}\right) \chi_{B_{2 R_{n}}(0)}+C\|u\|_{L^{\infty}\left(\mathbb{R}^{N}\right)} \frac{1-\varepsilon_{n}}{R_{n}} \chi_{B_{2 R_{n}}(0) \backslash B_{R_{n}}(0)}\right)^{p(x)}, \quad \forall x \in \mathbb{R}^{N} .
$$

Let $\varepsilon>0$ be arbitrary but fixed, and let $\delta(\varepsilon)>0$ be such that (5.4) holds. Using the previous estimates and (5.4) we find that for each $x \in \mathbb{R}^{N}$ we have

$$
\begin{aligned}
\left|\nabla v_{n}(x)\right|^{p(x) \leq} \leq & (1+\varepsilon)|\nabla u(x)|^{p(x)}\left(1-\varepsilon_{n}\right)^{p(x)} \chi_{B_{2 R_{n}}(0)}+ \\
& (1+\delta(\varepsilon))^{p^{+}}\left(C\|u\|_{L^{\infty}\left(\mathbb{R}^{N}\right)}\right)^{p(x)}\left(\frac{1-\varepsilon_{n}}{R_{n}}\right)^{p(x)} \chi_{B_{2 R_{n}}(0) \backslash B_{R_{n}}(0)} \leq \\
\leq & (1+\varepsilon)\left\||\nabla u|^{p(\cdot)}\right\|_{L^{\infty}\left(\mathbb{R}^{N}\right)}\left(1-\varepsilon_{n}\right) \chi_{B_{2 R_{n}}(0)}+ \\
& C_{\varepsilon}^{p^{+}}\left(\frac{1-\varepsilon_{n}}{R_{n}}\right)^{p^{-}} \chi_{B_{2 R_{n}}(0) \backslash B_{R_{n}}(0)} \\
= & (1+\varepsilon)\left\||\nabla u|^{p(\cdot)}\right\|_{L^{\infty}\left(\mathbb{R}^{N}\right)}\left(1-\varepsilon_{n}\right) \chi_{B_{R_{n}}(0)}+ \\
& {\left[(1+\varepsilon)\left\||\nabla u|^{p(\cdot)}\right\|_{L^{\infty}\left(\mathbb{R}^{N}\right)}\left(1-\varepsilon_{n}\right)+C_{\varepsilon}^{p^{+}}\left(\frac{1-\varepsilon_{n}}{R_{n}}\right)^{p^{-}}\right] \chi_{B_{2 R_{n}}(0) \backslash B_{R_{n}}(0)}, }
\end{aligned}
$$

where $C_{\varepsilon}:=(1+\delta(\varepsilon)) \max \left\{1, C\|u\|_{L^{\infty}\left(\mathbb{R}^{N}\right)}\right\}$. Next, we show that for $n \in \mathbb{N}$ sufficiently large we have

$$
(1+\varepsilon)\left\||\nabla u|^{p(\cdot)}\right\|_{L^{\infty}\left(\mathbb{R}^{N}\right)}\left(1-\varepsilon_{n}\right)+C_{\varepsilon}^{p^{+}}\left(\frac{1-\varepsilon_{n}}{R_{n}}\right)^{p^{-}} \leq(1+\varepsilon)\left\||\nabla u|^{p(\cdot)}\right\|_{L^{\infty}\left(\mathbb{R}^{N}\right)},
$$

or, equivalently,

$$
C_{\varepsilon}^{p^{+}}\left(\frac{1-\varepsilon_{n}}{R_{n}}\right)^{p^{-}} \leq(1+\varepsilon) \varepsilon_{n}\left\||\nabla u|^{p(\cdot)}\right\|_{L^{\infty}\left(\mathbb{R}^{N}\right)} .
$$

This inequality holds since

$$
\lim _{n \rightarrow \infty}(1+\varepsilon) \varepsilon_{n} R_{n}^{p^{-}}=\lim _{n \rightarrow \infty}(1+\varepsilon)\left(n p^{-}\right)^{-\frac{1}{4 N}}\left(n p^{-}\right)^{\frac{p^{-}}{2 N}}=C \lim _{n \rightarrow \infty} n^{\frac{p^{-}}{2 N}-\frac{1}{4 N}}=\infty,
$$

where here, and in what follows, $C>0$ is a real constant which may vary from line to line and expression to expression. Hence, for $x \in \mathbb{R}^{N}$ and $n \in \mathbb{N}$ sufficiently large, we have

$$
\left|\nabla v_{n}(x)\right|^{p(x)} \leq(1+\varepsilon)\left\||\nabla u|^{p(\cdot)}\right\|_{L^{\infty}\left(\mathbb{R}^{N}\right)} \chi_{B_{2 R_{n}}(0)},
$$

which gives

$$
\left|\nabla v_{n}(x)\right|^{n p(x)} \leq(1+\varepsilon)^{n}\left\||\nabla u|^{p(\cdot)}\right\|_{L^{\infty}\left(\mathbb{R}^{N}\right)}^{n} \chi_{B_{2 R_{n}}(0)} .
$$


It follows that

$$
\begin{aligned}
G_{n}\left(v_{n}\right) & =\left(\int_{\mathbb{R}^{N}} \frac{1}{n p(x)}\left|\nabla v_{n}(x)\right|^{n p(x)} d x\right)^{1 / n} \\
& \leq\left(\int_{\mathbb{R}^{N}} \frac{1}{n p(x)}(1+\varepsilon)^{n}\left\||\nabla u|^{p(\cdot)}\right\|_{L^{\infty}\left(\mathbb{R}^{N}\right)}^{n} \chi_{B_{2 R_{n}}(0)} d x\right)^{1 / n} \\
& \leq\left(\frac{1}{n p^{-}}\right)^{1 / n}(1+\varepsilon)\left\||\nabla u|^{p(\cdot)}\right\|_{L^{\infty}\left(\mathbb{R}^{N}\right)}\left|B_{2 R_{n}}(0)\right|^{1 / n} \\
& =\left(\frac{1}{n p^{-}}\right)^{1 / n}(1+\varepsilon)\left\||\nabla u|^{p(\cdot)}\right\|_{L^{\infty}\left(\mathbb{R}^{N}\right)}\left(C R_{n}^{N}\right)^{1 / n} \\
& =\left(\frac{1}{n p^{-}}\right)^{1 / n}(1+\varepsilon)\left\||\nabla u|^{p(\cdot)}\right\|_{L^{\infty}\left(\mathbb{R}^{N}\right)} C^{1 / n} n^{1 /(2 n)} .
\end{aligned}
$$

Since the right hand side converges to $(1+\varepsilon)\left\||\nabla u|^{p(\cdot)}\right\|_{L^{\infty}\left(\mathbb{R}^{N}\right)}$ as $n \rightarrow \infty$, we deduce that for each $\varepsilon>0$ we have

$$
\limsup _{n \rightarrow \infty} G_{n}\left(v_{n}\right) \leq(1+\varepsilon)\left\||\nabla u|^{p(\cdot)}\right\|_{L^{\infty}\left(\mathbb{R}^{N}\right)}
$$

Letting $\varepsilon \rightarrow 0$, we obtain

$$
G_{\infty}(u) \geq \limsup _{n \rightarrow \infty} G_{n}\left(v_{n}\right) .
$$

II. We now show that (5.2) holds. Let $\left\{n_{k}\right\}$ be a given subsequence of $\{k\}$ (clearly, $n_{k} \geq k$ ) and let $u_{k} \rightarrow u$ in $L^{2}\left(\mathbb{R}^{N}\right)$. Without loss of generality, we may assume that $u_{k} \in W^{1, n_{k} p(\cdot)}\left(\mathbb{R}^{N}\right) \cap L^{2}\left(\mathbb{R}^{N}\right)$ (which, in particular, implies that $\left|\nabla u_{k}\right|^{p(\cdot)} \in L^{n_{k}}\left(\mathbb{R}^{N}\right)$ ), and that

$$
\liminf _{k \rightarrow \infty} G_{n_{k}}\left(u_{k}\right)=\lim _{k \rightarrow \infty} G_{n_{k}}\left(u_{k}\right)=: L<\infty .
$$

We can cover the space $\mathbb{R}^{N}$ with a countable number of open sets $\Omega_{j}$ with $\left|\Omega_{j}\right|=1$ for each $j \in \mathbb{N}$, i.e.

$$
\mathbb{R}^{N}=\bigcup_{j=1}^{\infty} \Omega_{j}, \quad\left|\Omega_{j}\right|=1, \forall j \in \mathbb{N} .
$$

Fix $j \in \mathbb{N}$, and let $q \geq 1$ be arbitrary. For $k \in \mathbb{N}$ sufficiently large, we have

$$
\begin{aligned}
\int_{\Omega_{j}}\left|\nabla u_{k}(x)\right|^{q p^{+}} d x & \leq\left|\Omega_{j}\right|+\int_{\Omega_{j}}\left|\nabla u_{k}(x)\right|^{\frac{p(x) q p^{+}}{p^{-}}} d x \leq\left|\Omega_{j}\right|+\left(\int_{\Omega_{j}}\left|\nabla u_{k}(x)\right|^{n_{k} p(x)} d x\right)^{\frac{q p^{+}}{n_{k} p^{-}}}\left|\Omega_{j}\right|^{1-\frac{q p^{+}}{n_{k} p^{-}}} \\
& \leq 1+\left(n_{k} p^{+}\right)^{\frac{q p^{+}}{n_{k} p^{-}}} G_{n_{k}}\left(u_{k}\right)^{\frac{q p^{+}}{p^{-}}},
\end{aligned}
$$

where we have used Hölder's inequality. Thus, $\left\{\nabla u_{k}\right\}$ is bounded in $L^{q p^{+}}\left(\Omega_{j} ; \mathbb{R}^{N}\right)$. Since $u_{k} \rightarrow u$ weakly in $L^{2}\left(\mathbb{R}^{N}\right)$ and $u_{k} \rightarrow u$ in $L^{1}\left(\Omega_{j}\right)$, we deduce by Poincaré-Wirtinger's inequality that $\left\{u_{k}\right\}$ is bounded in $L^{q p^{+}}\left(\Omega_{j}\right)$. Thus, $\left\{u_{k}\right\}$ is bounded in $W^{1, q p^{+}}\left(\Omega_{j}\right)$. It follows that we can extract a subsequence (not relabelled) such that $u_{k} \rightarrow u$ weakly in $W^{1, q p^{+}}\left(\Omega_{j}\right)$. Since $p(x) \leq p^{+}$for any $x \in \Omega_{j}, W^{1, q p^{+}}\left(\Omega_{j}\right)$ is continuously embedded in $W^{1, q p(\cdot)}\left(\Omega_{j}\right)$, and we deduce that $u_{k} \rightarrow u$ weakly in $W^{1, q p(\cdot)}\left(\Omega_{j}\right)$. Then $[35$, Lemma 3.4] yields

$$
\int_{\Omega_{j}}|\nabla u(x)|^{q p(x)} d x \leq \liminf _{k \rightarrow \infty} \int_{\Omega_{j}}\left|\nabla u_{k}(x)\right|^{q p(x)} d x
$$


An alternative argument for (5.7) is as follows: Let $f: \Omega_{j} \times \mathbb{R}^{N} \rightarrow[0,+\infty)$ be defined by $f(x, v):=$ $|v|^{q p(x)}$. Note that $f$ is continuous, and that it satisfies the growth condition $0 \leq f(x, v) \leq C\left(1+|v|^{q p^{+}}\right)$ for all $(x, v) \in \Omega_{j} \times \mathbb{R}^{N}$. Also, $f(x, \cdot)$ is convex for all $x \in \Omega_{j}$. Since $u_{k} \rightarrow u$ weakly in $W^{1, q p^{+}}\left(\Omega_{j}\right),(5.7)$ now follows from well-known weak lower semicontinuity results for functionals of the form

$$
u \mapsto \int_{\Omega_{j}} f(x, \nabla u(x)) d x
$$

(see, e.g., [20]). Applying again Hölder's inequality, we find

$$
\int_{\Omega_{j}}\left|\nabla u_{k}\right|^{q p(x)} d x \leq\left(\int_{\Omega_{j}}\left|\nabla u_{k}\right|^{n_{k} p(x)} d x\right)^{\frac{q}{n_{k}}}\left|\Omega_{j}\right|^{1-\frac{q}{n_{k}}} \leq\left(n_{k} p^{+}\right)^{\frac{q}{n_{k}}} G_{n_{k}}\left(u_{k}\right)^{q}\left|\Omega_{j}\right|^{1-\frac{q}{n_{k}}} .
$$

Thus, taking into account (5.5),

$$
\limsup _{k \rightarrow \infty}\left(\int_{\Omega_{j}}\left|\nabla u_{k}\right|^{q p(x)} d x\right)^{\frac{1}{q}} \leq\left|\Omega_{j}\right|^{\frac{1}{q}} \liminf _{k \rightarrow \infty} G_{n_{k}}\left(u_{k}\right) .
$$

Finally, using the fact that

$$
\left(\liminf _{k \rightarrow \infty} \int_{\Omega_{j}}\left|\nabla u_{k}\right|^{q p(x)} d x\right)^{\frac{1}{q}} \leq \limsup _{k \rightarrow \infty}\left(\int_{\Omega_{j}}\left|\nabla u_{k}\right|^{q p(x)} d x\right)^{\frac{1}{q}}
$$

and in view of (5.7) and (5.8), we obtain that

$$
\left\||\nabla u|^{p(\cdot)}\right\|_{L^{q}\left(\Omega_{j}\right)} \leq\left|\Omega_{j}\right|^{\frac{1}{q}} \liminf _{k \rightarrow \infty} G_{n_{k}}\left(u_{k}\right) .
$$

Letting $q \rightarrow \infty$, we obtain

$$
\left\||\nabla u|^{p(\cdot)}\right\|_{L^{\infty}\left(\Omega_{j}\right)} \leq L .
$$

The above inequality holds for each $j \in \mathbb{N}$, and since $\mathbb{R}^{N}$ is a countable union of sets $\Omega_{j}$ we deduce that

$$
|\nabla u(x)|^{p(x)} \leq L \text { a.e. } x \in \mathbb{R}^{N} .
$$

Thus,

$$
\left\||\nabla u|^{p(\cdot)}\right\|_{L^{\infty}\left(\mathbb{R}^{N}\right)} \leq L=\liminf _{k \rightarrow \infty} G_{n_{k}}\left(u_{k}\right) .
$$

This concludes the proof of Theorem 3 .

\section{A MODEL FOR SANDPILES}

To identify the limit of the solutions $u_{n}$ of problem (1.5) (see the Introduction), we will use the methods of Convex Analysis, and so we must first recall some terminology (see [25], [15] and [5]).

If $H$ is a real Hilbert space with inner product $(\cdot, \cdot)$ and $\Psi: H \rightarrow(-\infty,+\infty]$ is convex, then the subdifferential of $\Psi$ is defined as the multivalued operator $\partial \Psi$ given by

$$
v \in \partial \Psi(u) \Longleftrightarrow \Psi(w)-\Psi(u) \geq(v, w-u) \quad \forall w \in H .
$$

Recall that the epigraph of $\Psi$ is defined by

$$
\operatorname{Epi}(\Psi)=\{(u, \lambda) \in H \times \mathbb{R}: \lambda \geq \Psi(u)\} .
$$


Given $K$ a closed convex subset of $H$, we define the indicator function of $K$ by

$$
I_{K}(u)= \begin{cases}0 & \text { if } u \in K, \\ +\infty & \text { if } u \notin K .\end{cases}
$$

Then the subdifferential is characterized by

$$
v \in \partial I_{K}(u) \Longleftrightarrow u \in K \text { and }(v, w-u) \leq 0 \quad \forall w \in K .
$$

When the convex functional $\Psi: H \rightarrow(-\infty,+\infty]$ is proper, lower-semicontinuous, and such that $\min \Psi=0$, it is well known (see [15]) that the abstract Cauchy problem

$$
\left\{\begin{array}{l}
u_{t}+\partial \Psi(u) \ni f, \quad \text { a.e } t \in(0, T), \\
u(0)=u_{0},
\end{array}\right.
$$

has a unique solution for any $f \in L^{2}(0, T ; H)$ and $u_{0} \in \overline{D(\partial \Psi)}$.

The Mosco convergence is a very useful tool to study convergence of solutions of parabolic problems. The following theorem is a consequence of results in [16] and [5].

Theorem 4. Let $\Psi_{n}, \Psi: H \rightarrow(-\infty,+\infty]$ be convex and lower semicontinuous functionals. Then the following statements are equivalent:

(i) $\Psi_{n}$ converges to $\Psi$ in the sense of Mosco.

(ii) $\left(I+\lambda \partial \Psi_{n}\right)^{-1} u \rightarrow(I+\lambda \partial \Psi)^{-1} u \quad \forall \lambda>0, u \in H$.

Moreover, either one of the above conditions, $(i)$ or (ii), imply that

(iii) for every $u_{0} \in \overline{D(\partial \Psi)}$ and $u_{0, n} \in \overline{D\left(\partial \Psi_{n}\right)}$ such that $u_{0, n} \rightarrow u_{0}$, and for every $f_{n}, f \in L^{1}(0, T ; H)$ with $f_{n} \rightarrow f$, if $u_{n}(t), u(t)$ are solutions of the abstract Cauchy problems

$$
\left\{\begin{array}{l}
\left(u_{n}\right)_{t}+\partial \Psi_{n}\left(u_{n}\right) \ni f_{n} \quad \text { a.e. } t \in(0, T) \\
u_{n}(0)=u_{0, n}
\end{array}\right.
$$

and

$$
\left\{\begin{array}{l}
u_{t}+\partial \Psi(u) \ni f \quad \text { a.e. } t \in(0, T) \\
u(0)=u_{0},
\end{array}\right.
$$

respectively, then

$$
u_{n} \rightarrow u \quad \text { in } C([0, T]: H)
$$

Now we observe that if we take $H=L^{2}\left(\mathbb{R}^{N}\right)$ and

$$
\Psi_{n}(u):= \begin{cases}\int_{\mathbb{R}^{N}} \frac{\lambda(x)^{n}}{n p(x)}|\nabla u(x)|^{n p(x)} d x & \text { if } u \in L^{2}\left(\mathbb{R}^{N}\right) \cap W^{1, n p(\cdot)}\left(\mathbb{R}^{N}\right) \\ +\infty & \text { otherwise, }\end{cases}
$$

then the associated PDE reads:

$$
\left\{\begin{array}{l}
\left(u_{n}\right)_{t}+\operatorname{div}\left(\lambda(x)^{n}\left|\nabla u_{n}\right|^{n p(x)-2} \nabla u_{n}\right)=f \quad \text { a.e. } t \in(0, T) \\
u_{n}(0)=u_{0} .
\end{array}\right.
$$


In this case, in view of our Theorem 2, together with Theorem 4, we deduce that

$$
u_{n} \rightarrow u \quad \text { in } C\left([0, T]: L^{2}\left(\mathbb{R}^{N}\right)\right),
$$

where $u$ is the solution to

$$
\left\{\begin{array}{l}
u_{t}+\partial \Psi_{\infty}(u) \ni f \quad \text { a.e. } t \in(0, T) \\
u(0)=u_{0},
\end{array}\right.
$$

and $\Psi_{\infty}: L^{2}\left(\mathbb{R}^{N}\right) \rightarrow[0,+\infty]$ is given by

$$
\Psi_{\infty}(u)= \begin{cases}0 & \text { if } \lambda(x)|\nabla u(x)|^{p(x)} \leq 1 \text { a.e. } x \in \mathbb{R}^{N} \\ +\infty & \text { otherwise. }\end{cases}
$$

As already mentioned in the Introduction, this limit can be seen as a model for the growth of a sandpile in which the critical slope of the sand depends explicitly on the spatial location. This dependence can be explained by differences in the sand composition or humidity.

We present below, in the one-dimensional case $N=1$, some explicit examples of solutions to the limiting evolution problem in the particular case where $f=\delta_{0}$ and $u_{0}=0$, subject to pointwise constraints on the derivatives.

First, let us consider the case in which the restriction on the derivative reads as

$$
\left|u_{x}\right|(x) \leq 1 \quad \text { for } x \leq 0, \quad \text { and } \quad\left|u_{x}\right|(x) \leq 1 / 2 \quad \text { for } x>0,
$$

that is,

$$
\left|u_{x}(x)\right| \leq A(x):= \begin{cases}1 & \text { if } x \leq 0 \\ 1 / 2 & \text { if } x>0 .\end{cases}
$$

Now, let

$$
u(x, t)= \begin{cases}x+z(t) & \text { if } \quad 0>x>-z(t) \\ z(t)-\frac{1}{2} x & \text { if } \quad 2 z(t)>x \geq 0 \\ 0 & \text { otherwise, }\end{cases}
$$

with $z(t)=\sqrt{\frac{2 t}{3}}$ the solution to

$$
z^{\prime}(t)=\frac{1}{3 z(t)}, \quad z(0)=0 .
$$

The function $u(x, t)$ defined by $(6.1)$ is the unique solution to the problem

$$
\left\{\begin{array}{l}
u_{t}+\partial \Psi_{\infty}(u) \ni \delta_{0} \quad \text { a.e. } t \in(0, T) \\
u(0)=0
\end{array}\right.
$$

where $\Psi_{\infty}: L^{2}(\mathbb{R}) \rightarrow[0,+\infty]$ is given by

$$
\Psi_{\infty}(u)= \begin{cases}0 & \text { if }\left|u^{\prime}(x)\right| \leq A(x) \text { a.e. } x \in \mathbb{R} \\ +\infty & \text { otherwise. }\end{cases}
$$

Let us prove this fact. We need to show that for every $v \in L^{2}(\mathbb{R})$ we have

$$
\Psi_{\infty}(v) \geq \Psi_{\infty}(u(\cdot, t))+\int_{\mathbb{R}}\left(\delta_{0}-u_{t}(\cdot, t)\right)(v-u(\cdot, t)) d x .
$$


As $\left|u_{x}(x, t)\right| \leq A(x), x \in \mathbb{R}$, we have $\Psi_{\infty}(u(\cdot, t))=0$, and thus we can restrict our attention to functions $v \in L^{2}(\mathbb{R})$ such that $\Psi_{\infty}(v)=0$, that is, $\left|v^{\prime}(x)\right| \leq A(x)$ (otherwise $\Psi_{\infty}(v)=+\infty$, and there is nothing to prove). Hence, we are left with

$$
\int_{\mathbb{R}} u_{t}(x, t)(v(x)-u(x, t)) d x \geq v(0)-u(0, t) .
$$

Now, since

$$
u_{t}(x, t)= \begin{cases}z^{\prime}(t) & \text { if }-z(t) \leq x \leq 2 z(t) \\ 0 & \text { otherwise }\end{cases}
$$

we need to verify:

$$
\int_{0}^{2 z(t)}(v(x)-u(x, t)) d x+\int_{-z(t)}^{0}(v(x)-u(x, t)) d x \geq 3 z(t)(v(0)-u(0, t)) .
$$

We will show that

$$
\int_{0}^{2 z(t)}(v(x)-u(x, t)) d x \geq 2 z(t)(v(0)-u(0, t))
$$

and

$$
\int_{-z(t)}^{0}(v(x)-u(x, t)) d x \geq z(t)(v(0)-u(0, t))
$$

hold. The fact that (6.3) or, equivalently,

$$
\int_{0}^{2 z(t)}(v(0)-v(x)) d x \leq \int_{0}^{2 z(t)}(u(0, t)-u(x, t)) d x
$$

holds follows by taking into account the fact that $v$ satisfies $\left|v^{\prime}(x)\right| \leq A(x)=\frac{1}{2}$ for $x \in(0,2 z(t))$, and observing that this gives

$$
u(0, t)-u(x, t)=\frac{1}{2} x \geq v(0)-v(x) \quad \text { for } x \in(0,2 z(t)) .
$$

Similarly, one can also show (6.4). We conclude that $u(x, t)$ given by (6.1) solves (6.2).

The above discussion can be adapted to treat the general case where $A$ is only required to satisfy

$$
0<c_{1} \leq A(x) \leq c_{2}<+\infty .
$$

In this case, for every $z>0$ there exists $s_{-}(z)<0$ and $s_{+}(z)>0$ such that

$$
\int_{s_{-}(z)}^{0} A(s) d s+z=0, \text { and }-\int_{0}^{s_{+}(z)} A(s) d s+z=0 .
$$

The solution $u=u(x, t)$ to $(6.2)$ (we keep the data $f=\delta_{0}$ and $u_{0}=0$ ) is now given by

$$
u(x, t)= \begin{cases}\int_{0}^{x} A(s) d s+z(t) & \text { if } s_{-}(z(t)) \leq x \leq 0 \\ -\int_{0}^{x} A(s) d s+z(t) & \text { if } 0<x \leq s_{+}(z(t)) \\ 0 & \text { otherwise, }\end{cases}
$$

with $z=z(t)$ being the solution to the problem

$$
z^{\prime}(t)=\frac{1}{s_{+}(z(t))-s_{-}(z(t))}, \quad z(0)=0 .
$$


To prove this, we need to show, as before, that

$$
\Psi_{\infty}(v) \geq \Psi_{\infty}(u(\cdot, t))+\int_{\mathbb{R}}\left(\delta_{0}-u_{t}(\cdot, t)\right)(v-u(\cdot, t)) d x \forall v \in L^{2}(\mathbb{R}) .
$$

Since $\left|u_{x}(x, t)\right| \leq A(x)$, we have $\Psi_{\infty}(u(\cdot, t))=0$, and thus, without loss of generality, we only consider functions $v \in L^{2}(\mathbb{R})$ such that $\Psi_{\infty}(v)=0$, that is, $\left|v^{\prime}(x)\right| \leq A(x)$ (otherwise $\Psi_{\infty}(v)=+\infty$, and there is nothing to prove). Hence, we need to show that

$$
\int_{\mathbb{R}} u_{t}(x, t)(v(x)-u(x, t)) d x \geq v(0)-u(0, t) .
$$

Since

$$
u_{t}(x, t)= \begin{cases}z^{\prime}(t) & s_{-}(z(t)) \leq x \leq s_{+}(z(t)), \\ 0 & \text { otherwise }\end{cases}
$$

this reduces to showing that

$$
\int_{0}^{s_{+}(z(t))}(v(x)-u(x, t)) d x+\int_{s_{-}(z(t))}^{0}(v(x)-u(x, t)) d x \geq\left(s_{+}(z(t))-s_{-}(z(t))\right)(v(0)-u(0, t)) .
$$

To this aim, it is enough to prove that

$$
\int_{0}^{s_{+}(z(t))}(v(x)-u(x, t)) d x \geq s_{+}(z(t))(v(0)-u(0, t)),
$$

and

$$
\int_{s_{-}(z(t))}^{0}(v(x)-u(x, t)) d x \geq-s_{-}(z(t))(v(0)-u(0, t))
$$

hold. Using the fact that $v$ satisfies $\left|v^{\prime}(x)\right| \leq A(x)$ for $x \in\left(0, s_{+}(z(t))\right.$, we have

$$
u(0, t)-u(x, t)=\int_{0}^{x} A(s) d s \geq v(0)-v(x) \quad \text { for all } x \in\left(0, s_{+}(z(t)),\right.
$$

which implies that

$$
\int_{0}^{s_{+}(z(t))}(v(0)-v(x)) d x \leq \int_{0}^{s_{+}(z(t))}(u(0, t)-u(x, t)) d x
$$

This shows that (6.7) holds. The remaining inequality, (6.8), follows similarly. We conclude that $u=u(x, t)$ given by (6.6) is the solution to (6.2) for functions $A$ satisfying (6.5).

Finally, let us consider a nontrivial initial condition $u_{0}=u_{0}(x)$ satisfying the restriction $\left|\left(u_{0}\right)^{\prime}(x)\right| \leq$ $A(x)$. We assume again that (6.5) holds, and we keep the data $f=\delta_{0}$.

In this case, for every $z>u_{0}(0)$ there exists $s_{-}(z)<0$ and $s_{+}(z)>0$ such that

$$
\int_{s_{-}(z)}^{0} A(s) d s+z=u_{0}\left(s_{-}(z)\right), \quad \text { and } \quad-\int_{0}^{s_{+}(z)} A(s) d s+z=u_{0}\left(s_{+}(z)\right) .
$$

The solution $u=u(x, t)$ to $(6.2)$ (with $\left.u(0)=u_{0}\right)$ is given by $(6.6)$, that is,

$$
u(x, t)= \begin{cases}\int_{0}^{x} A(s) d s+z(t) & \text { if } s_{-}(z(t)) \leq x \leq 0, \\ -\int_{0}^{x} A(s) d s+z(t) & \text { if } 0<x \leq s_{+}(z(t)), \\ 0 & \text { otherwise, }\end{cases}
$$


but now $z=z(t)$ is the solution to the ODE

$$
z^{\prime}(t)=\frac{1}{s_{+}(z(t))-s_{-}(z(t))}, \quad z(0)=u_{0}(0) .
$$

To prove this, we need to show, arguing as before, that

$$
\int_{\mathbb{R}} u_{t}(x, t)(v(x)-u(x, t)) d x \geq v(0)-u(0, t) .
$$

Since

$$
u_{t}(x, t)= \begin{cases}z^{\prime}(t) & s_{-}(z(t)) \leq x \leq s_{+}(z(t)) \\ 0 & \text { otherwise, }\end{cases}
$$

this reduces to showing that

$$
\int_{0}^{s_{+}(z(t))}(v(x)-u(x, t)) d x+\int_{s_{-}(z(t))}^{0}(v(x)-u(x, t)) d x \geq\left(s_{+}(z(t))-s_{-}(z(t))\right)(v(0)-u(0, t)) .
$$

The proof of this fact runs exactly as before, we show that (6.7) and (6.8) hold, following the same steps performed in the case $u_{0}=0$. We conclude that $u=u(x, t)$ given by $(6.9)$ is the solution to (6.2).

Acknowledgements. The research of M. Bocea was partially supported by the U.S. National Science Foundation under Grant No. DMS-0806789. The research of M. Mihăilescu was partially supported by the grant CNCSIS-UEFISCSU, project number PN II-RU PD-117/2010 "Probleme neliniare modelate de operatori diferenţiali neomogeni". M. Perez-Llanos partially supported by the Fundação para a Ciência e a Tecnologia (Portugal) and project UTAustin/MAT/0057/2008. J. D. Rossi partially supported by Ministerio de Ciencia e Innovacion grant MTM2008-05824, Spain, and by UBA X066 and CONICET, Argentina.

\section{REFERENCES}

[1] E. Acerbi and G. Mingione, Regularity results for stationary electro-rheological fluids, Arch. Ration. Mech. Anal., 164 (2002), 213-259.

[2] E. Acerbi and G. Mingione, Gradient estimates for the $p(x)$-Laplacean system, J. Reine Angew. Math., 584 (2005), $117-148$.

[3] F. Andreu, J. M. Mazon, J. D. Rossi and J. Toledo, The limit as $p \rightarrow \infty$ in a nonlocal p-Laplacian evolution equation. A nonlocal approximation of a model for sandpiles. Calc. Var. Partial Differential Equations, 35(3), (2009), $279-316$.

[4] G. Aronsson, L. C. Evans and Y. Wu, Fast/slow diffusion and growing sandpiles. J. Differential Equations, 131 (1996), 304-335.

[5] H. Attouch, Familles d'opérateurs maximaux monotones et mesurabilité. Ann. Mat. Pura Appl., 120 (1979), 35-111.

[6] H. Attouch, Variational convergence for functions and operators, Pitman, London, 1984.

[7] M. Bocea and V. Nesi, $\Gamma$-convergence of power-law functionals, variational principles in $L^{\infty}$, and applications, SIAM J. Math. Anal. 39 (2008), 1550-1576.

[8] M. Bocea, M. Mihăilescu, and C. Popovici, On the asymptotic behavior of variable exponent power-law functionals and applications, In Press: Ricerche Mat. 59 No. 2 (2010) DOI: 10.1007/s11587-010-0081-x.

[9] Ph. Benilan and M.G. Crandall, Completely Accretive Operators, in Semigroups Theory and Evolution Equations, Ph. Clement et al. editors, Marcel Dekker, 1991, pp. 41-76.

[10] P. Cannarsa, P. Cardaliaguet and E. Giorgieri, Hölder regularity of the normal distance with an application to a PDE model for growing sandpiles. Trans. Amer. Math. Soc. 359 (2007), no. 6, 2741-2775.

[11] P. Cannarsa, P. Cardaliaguet and C. Sinestrari, On a differential model for growing sandpiles with non-regular sources. Comm. Partial Differential Equations 34 (2009), no. 7-9, 656-675.

[12] Ph. Bénilan, M. G. Crandall and A. Pazy, Evolution Equations Governed by Accretive Operators. Book to appear. 
[13] Ph. Bénilan, L.C. Evans and R.F. Gariepy, On some singular limits of homogeneous semigroups, J. Evol. Equ. 3, (2003), 203-214.

[14] H. Brezis, Équations et inéquations non linéaires dans les espaces vectoriels en dualité, Ann. Inst. Fourier, 18, (1968), $115-175$.

[15] H. Brezis, Opérateur Maximaux Monotones et Semi-groupes de Contractions dans les Espaces de Hilbert, NorthHolland, 1973.

[16] H. Brezis and A. Pazy, Convergence and approximation of semigroups of nonlinear operators in Banach spaces, J. Functional Analysis 9 (1972), 63-74.

[17] M. Camar-Eddine and P. Seppecher, Closure of the set of diffusion functionals with respect to the Mosco-convergence, Math. Models Methods Appl. Sci. 12 No. 8 (2002), 1153-1176.

[18] M. Camar-Eddine and P. Seppecher, Determination of the closure of the set of elasticity functionals, Arch. Ration. Mech. Anal. 170 No. 3 (2003), 211-245.

[19] Y. Chen, S. Levine and M. Rao, Variable exponent, linear growth functionals in image processing, SIAM J. Appl. Math. 66 No. 4 (2006), 1383-1406.

[20] B. Dacorogna, Direct methods in the calculus of variations. Applied mathematical sciences, vol. 78. Berlin, Germany: Springer, 1989.

[21] E. De Giorgi, Sulla convergenza di alcune succesioni di integrali del tipo dell'area, Rend. Mat. 8 (1975), $277-294$.

[22] E. De Giorgi and T. Franzoni, Su un tipo di convergenza variazionale, Atti Accad. Naz. Lincei Rend. Cl. Sci. Fis. Mat. Natur. 58 (1975), 842-850.

[23] L. Diening, P. Hästö and A. Nekvinda, Open problems in variable exponent Lebesgue and Sobolev spaces, in: FSDONA04 Proceedings, Drabek and Rakosnik (eds.), pp. 38-58, Milovy, Czech Republic, 2005.

[24] J. Dorfman and L. C. Evans, "lakes and rivers" heuristic metaphor for the singular limit of a nonlinear diffusion PDE. SIAM J. Math. Anal. 41 (2009), no. 4, 1621-1652.

[25] H. I. Ekeland and R. Temam, Convex Analysis and Variational Problems, North-Holland, 1972.

[26] L. C. Evans, M. Feldman and R. F. Gariepy, Fast/slow diffusion and collapsing sandpiles, J. Differential Equations 137 (1997), 166-209.

[27] L. C. Evans and Fr. Rezakhanlou, A stochastic model for growing sandpiles and its continuum limit, Comm. Math. Phys. 197 (1998), 325-345.

[28] M. Falcone and S. Finzi Vita, A finite-difference approximation of a two-layer system for growing sandpiles, SIAM J. Sci. Comput., 28 (2006), 1120-1132.

[29] M. Falcone and S. Finzi Vita, A numerical study for growing sandpiles on flat tables with walls, in Systems, Control, Modeling, and Optimization, IFIP Int. Fed. Inf. Process 202, Springer, New York, 2006, pp. 127-137.

[30] M. Feldman, Growth of a sandpile around an obstacle. Monge Ampère equation: applications to geometry and optimization (Deerfield Beach, FL, 1997), 55-78, Contemp. Math., 226, Amer. Math. Soc., Providence, RI, 1999.

[31] A. Garroni, V. Nesi and M. Ponsiglione, Dielectric breakdown: optimal bounds, Proc. R. Soc. Lond. A 457 (2001), $2317-2335$

[32] P. Harjulehto, P. Hästö, Ú. V. Lê and M. Nuortio, Overview of differential equations with non-standard growth, Nonlinear Anal. 72 (2010), 4551-4574.

[33] O. Kováčik and J. Rákosník, On spaces $L^{p(x)}$ and $W^{1, p(x)}$, Czechoslovak Math. J. 41 (116) (1991), 592-618.

[34] M. B. Lignola and J. Morgan, Convergence of solutions of quasi-variational inequalities and applications, Topological Methods in Nonlinear Analysis, Journal of the Juliusz Schauder Center, 10 (1997), 375-385.

[35] M. Mihăilescu and V. Rădulescu, A multiplicity result for a nonlinear degenerate problem arising in the theory of electrorheological fluids, Proc. R. Soc. Lond. A 462 (2006), 2625-2641.

[36] U. Mosco, Approximation of the solutions of some variational inequalities, Ann. Sc. Norm. Super. Pisa Cl. Sci. 21 (1967), 373-394.

[37] U. Mosco, Convergence of convex sets and solutions of variational inequalities, Adv. Math 3 (1969), 510-585.

[38] U. Mosco, Composite media and asymptotic Dirichlet forms, J. Funct. Anal. 123 (1994), 368-421.

[39] O. V. Pugachev, On Mosco Convergence of Diffusion Dirichlet Forms, Theory Probab. Appl. 53 (2) (2009), $242-255$.

[40] L. Prigozhin, Sandpiles and river networks: Extended systems with nonlocal interactions, Phys. Rev. E., 49 (1994), 1161-1167.

[41] L. Prigozhin and B. Zaltzman, On the approximation of the dynamics of sandpile surfaces, Port. Math., 60 (2003), 127-137.

[42] K.R. Rajagopal and M. Ružička, Mathematical modelling of electrorheological fluids, Contin. Mech. Thermodyn. 13 (2001), 59-78.

[43] M. Ružička, Flow of shear dependent electrorheological fluids: unsteady space periodic case, Applied Nonlinear Analysis, 485-504, Kluwer/Plenum, New York, 1999. 
[44] M. Ružička, Electrorheological Fluids: Modeling and Mathematical Theory, Springer-Verlag, Berlin, 2002.

[45] A. A. Tolstonogov, Mosco convergence of integral functionals and its applications, Mat. Sb. 200 (3) (2009), 119-146.

\section{Marian Bocea}

Department of Mathematics, North Dakota State University

NDSU DEPT. \# 2750, P.O. Box 6050

FARGO, ND 58108-6050 U.S.A.

E-mail address: marian.bocea@ndsu.edu

Mihai MihăILESCU

Department of Mathematics, University of Craiova, 200585 Craiova, ROMANIA.

and

Department of Mathematics, Central European University, 1051 BudAPEST, HUNGARY

E-mail address: mmihailes@yahoo.com

Mayte PÉrez-Llanos

Department of Mathematics, University of Texas at Austin

1 University Station C1200, Austin, Texas 78712. U.S.A.

E-mail address: mayte@math.utexas.edu

Julio D. Rossi

Departamento de Análisis Matemático, Universidad de Alicante,

Ap. Correos 99, 03080 Alicante, SPAIN.

On leave from

Departamento de Matemática, FCEyn UBA,

Ciudad Universitaria, Pab 1 (1428),

Buenos Aires, ARGENTINA.

E-mail address: julio.rossi@ua.es 